\title{
THE SEMI-GROUP PROPERTY OF POISSON TRANSFORMATION AND SNOW'S INVERSION FORMULA
}

\section{HARRY POLLARD ${ }^{1}$}

To Einar Hille, on the occasion of his retirement from Yale University, June, 1962.

1. The Poisson transform. By the set $F$ we shall mean the family of functions $f$ which are integrable on each finite interval of the real axis and for which the integrals

$$
\int^{\infty} \frac{f(x)}{x^{2}} d x \text { and } \int_{-\infty} \frac{f(x)}{x^{2}} d x
$$

converge. For each member $f$ of this set and each positive number $a$ the Poisson transform $P_{a} f$ is defined by the point function

$$
\left(P_{a} f\right)(x)=\frac{1}{\pi} \int_{-\infty}^{\infty} \frac{a f(\xi)}{(x-\xi)^{2}+a^{2}} d \xi, \quad-\infty<x<\infty,
$$

which under condition (1) converges uniformly in each interval $0<a_{1} \leqq a \leqq a_{2}$, so that for fixed $x,\left(P_{a} f\right)(x)$ is continuous in $a>0$.

The semi-group property, easy to prove in case the integrals (1) converge absolutely, states that

$$
P_{b} P_{a} f=P_{a+b} f, \quad a>0, b>0 .
$$

The main purpose of this paper is to establish the validity of this property for each $f$ in $F$, thus removing the requirement of absolute convergence.

As an application it will be established in the last section of the paper that Snow's inversion formula for the integral transform

$$
g(x)=\frac{1}{\pi} \int_{-\infty}^{\infty} \frac{f(\xi)}{(x-\xi)^{2}+1} d \xi
$$

holds on the sole assumption that the integrals (1) converge. His own conditions are more restrictive (for a discussion and reference see $[1$, p. 94]).

2. Proof of the semi-group property. Let us denote by $k(x, a)$ the function

Received by the editors March 7, 1962.

1 Prepared under Air Force Contract No. AF 18(600)-685. 


$$
k(x, a)=\frac{1}{\pi} \frac{a}{x^{2}+a^{2}}
$$

The formula (2) states that

$$
\begin{array}{r}
\int_{-\infty}^{\infty} k(x-\xi, b) d \xi \int_{-\infty}^{\infty} k(\xi-y, a) f(y) d y=\int_{-\infty}^{\infty} k(x-y, a+b) f(y) d y, \\
a>0, b>0 .
\end{array}
$$

In view of the familiar fact that $k(x, a) * k(x, b)=k(x, a+b)$, the proof amounts to showing that

$$
\begin{aligned}
\lim _{R, S \rightarrow \infty} \frac{1}{\pi^{2}} \int_{-S}^{R} \frac{b d \xi}{(x-\xi)^{2}+b^{2}} \int_{-\infty}^{\infty} \frac{a f(y) d y}{(\xi-y)^{2}+a^{2}} \\
=\frac{1}{\pi^{2}} \int_{-\infty}^{\infty} f(y) d y \int_{-\infty}^{\infty} \frac{a b d \xi}{\left[(x-\xi)^{2}+b^{2}\right]\left[(\xi-y)^{2}+a^{2}\right]} .
\end{aligned}
$$

The inside integral on the left-hand side converges uniformly on finite intervals $-S \leqq \xi \leqq R$. This permits the interchange of the iterated integrals on the left-hand side, so we must show that the difference between

$$
\int_{-\infty}^{\infty} f(y) d y \int_{-S}^{R} \cdots d \xi
$$

and the right-hand side approaches zero as $R \rightarrow \infty, S \rightarrow \infty$. By $\cdots$ we mean

$$
\frac{a b}{\left[(x-\xi)^{2}+b^{2}\right]\left[(\xi-y)^{2}+a^{2}\right]} .
$$

This difference can be written

$$
\int_{-\infty}^{\infty} f(y) d y \int_{R}^{\infty} \cdots d \xi+\int_{-\infty}^{\infty} f(y) d y \int_{-\infty}^{-s} \cdots d \xi,
$$

where the convergence of each of the double integrals follows from subsequent considerations.

We shall show that as $R \rightarrow \infty$ the first of these iterated integrals vanishes; the proof that the second does as $S \rightarrow \infty$ is similar.

Let $\Delta(x, y)$ denote the expression defined by

$$
\begin{aligned}
\Delta & \equiv(x-y)^{4}+2\left(b^{2}+a^{2}\right)(x-y)^{2}+\left(b^{2}-a^{2}\right)^{2} \\
& =\left[(x-y)^{2}+(b+a)^{2}\right]\left[(x-y)^{2}+(b-a)^{2}\right] .
\end{aligned}
$$

Then by standard methods of integration 
(6)

$$
\begin{aligned}
\int_{-\infty}^{\infty} f(y) d y \int_{R}^{\infty} \cdots d \xi \\
=\int_{-\infty}^{\infty} f(y)\left(\frac{\pi}{2}-\arctan \frac{R-x}{b}\right) \frac{a}{\Delta}\left[(x-y)^{2}-b^{2}+a^{2}\right] d y \\
\quad+\int_{-\infty}^{\infty} f(y)\left(\frac{\pi}{2}-\arctan \frac{R-y}{a}\right) \frac{b}{\Delta}\left[(x-y)^{2}+b^{2}-a^{2}\right] d y \\
\quad-\int_{-\infty}^{\infty} f(y) \frac{a b}{\Delta}(y-x) \log \frac{(R-x)^{2}+b^{2}}{(R-y)^{2}+a^{2}} d y .
\end{aligned}
$$

The independent convergence of each of these three integrals on the right follows from the convergence of the integrals (1).

As $R \rightarrow \infty$ the first of these three approaches zero since the factor $\pi / 2-\operatorname{arc} \tan ((R-x) / b)$ can be moved outside the integral.

The second integral can be written

$$
I \equiv \int_{-\infty}^{\infty}\left(\frac{\pi}{2}-\arctan \frac{R-y}{a}\right) d G(y),
$$

where

$$
G(y)=-\int_{y}^{\infty} \frac{b}{\Delta}\left[(x-\eta)^{2}+b^{2}-a^{2}\right] f(\eta) d \eta, \quad \Delta=\Delta(x, \eta) .
$$

On integrating by parts we find that the integrated parts vanish, leaving

$$
I=\int_{-\infty}^{\infty} G(y) \frac{a}{(R-y)^{2}+a^{2}} d y .
$$

Consequently,

$$
I=\int_{-\infty}^{\infty} G(R-u) \frac{a}{u^{2}+a^{2}} d u .
$$

This vanishes as $R \rightarrow \infty$ because $G(\infty)=0$ and $G$ is a bounded function.

The third integral on the right-hand side of (6) can be written as

$$
J \equiv \int_{-\infty}^{\infty} \frac{y-x}{(x-y)^{2}+(b-a)^{2}} \log \frac{(R-x)^{2}+b^{2}}{(R-y)^{2}+a^{2}} d F(y),
$$

where

$$
F(y)=-\int_{-\infty}^{y} \frac{a b f(\eta) d \eta}{(x-\eta)^{2}+(a+b)^{2}}
$$


This follows from (5). Integration by parts yields

$$
\begin{aligned}
J \equiv & \int_{-\infty}^{\infty} F(y) \frac{\partial}{\partial y}\left\{\frac{y-x}{(x-y)^{2}+(b-a)^{2}} \log \frac{(R-x)^{2}+b^{2}}{(R-y)^{2}+a^{2}}\right\} d y \\
= & 2 \int_{-\infty}^{\infty} F(y) \frac{y-x}{(x-y)^{2}+(b-a)^{2}} \frac{R-y}{(R-y)^{2}+a^{2}} d y \\
& +\int_{-\infty}^{\infty} F(y) \frac{(b-a)^{2}-(y-x)^{2}}{\left[(x-y)^{2}+(b-a)^{2}\right]^{2}} \log \frac{(R-x)^{2}+b^{2}}{(R-y)^{2}+a^{2}} d y .
\end{aligned}
$$

The function $F(y)$ is bounded. Consequently the first integral on the right-hand side of (7) is bounded by a constant independent of $R$ times

$$
\int_{-\infty}^{\infty} \frac{1}{1+|y|} \frac{1}{1+|R-y|} d y .
$$

This can be computed to show that it vanishes as $R \rightarrow \infty$.

As for the last integral, it is dominated by a constant independent of $R$ times

$$
\int_{-\infty}^{\infty} \frac{1}{1+(x-y)^{2}}\left|\log \frac{(R-x)^{2}+b^{2}}{(R-y)^{2}+a^{2}}\right| d y .
$$

Let $y-x=\eta, R-x=T$. It remains only to show

$$
\lim _{T \rightarrow \infty} \int_{-\infty}^{\infty} \frac{1}{1+\eta^{2}}\left|\log \frac{T^{2}+b^{2}}{(T-\eta)^{2}+a^{2}}\right| d \eta=0 .
$$

The integral here is

$$
\begin{aligned}
\leqq & \int_{-\infty}^{\infty} \frac{1}{1+\eta^{2}}\left|\log \frac{T^{2}+a^{2}}{(T-\eta)^{2}+a^{2}}\right| d \eta \\
& +\int_{-\infty}^{\infty} \frac{1}{1+\eta^{2}}\left|\log \frac{T^{2}+b^{2}}{T^{2}+a^{2}}\right| d \eta
\end{aligned}
$$

of which the latter clearly vanishes as $T \rightarrow \infty$.

The first integral of (9) can be written

$$
\int_{-\infty}^{\infty}\left|\log \frac{T^{2}+a^{2}}{(T-\eta)^{2}+a^{2}}\right| d G(\eta)
$$

where $G(\eta)=\arctan \eta-(\pi / 2) \operatorname{sgn} \eta$. Integration by parts shows that this integral is numerically less than

$$
2 \int_{-\infty}^{\infty}|G(\eta)| \frac{|T-\eta|}{(T-\eta)^{2}+a^{2}} d \eta
$$


Since $|G(\eta)| \leqq A /(1+|\eta|)$ this integral is less than a constant (independent of $T)$, times

$$
\int_{-\infty}^{\infty} \frac{1}{1+|\eta|} \frac{1}{1+|T-\eta|} d \eta
$$

This vanishes as $T \rightarrow \infty$ for the reason given for (8).

The proof of (3) is now complete.

3. Snow's inversion formula for the transform (4). On the assumption that the integrals (1) converge, the integral (4) converges for $-\infty<x<\infty$ and admits analytic continuation into the region $|I z|$ $<1$, where $z=x+i y[2$, p. 543]. Under severe restrictions Snow [1] has shown that (4) is inverted by

$$
f(x)=\lim _{y \rightarrow 1}[g(x+i y)+g(x-i y)]-\left(P_{1} g\right)(x) .
$$

We show that this holds for almost all $x$ under the sole assumption that the integrals (1) converge. First by the continuity of $\left(P_{y} f\right)(x)$ as a function of $y$ the formula can be written as

$$
f(x)=\lim _{y \rightarrow 1}\left[g(x+i y)+g(x-i y)-\left(P_{y} g\right)(x)\right] .
$$

Next it follows from (4) that

$$
\begin{aligned}
& g(x+i y)+g(x-i y) \\
& \quad=\frac{1}{\pi} \int_{-\infty}^{\infty}\left[\frac{1}{(x+i y-\xi)^{2}+1}+\frac{1}{(x-i y-\xi)^{2}+1}\right] f(\xi) d \xi .
\end{aligned}
$$

Now $g=P_{1} f$ so that by the semi-group property

$$
P_{\mathrm{y}} g=P_{\mathrm{y}} P_{1} f=P_{\mathrm{y}+1} f \text {. }
$$

Hence,

$$
\left(P_{y} g\right)(x)=\frac{1}{\pi} \int_{-\infty}^{\infty} \frac{(1+y)}{(x-\xi)^{2}+(y+1)^{2}} d \xi .
$$

Subtract (12) from (11). By the identity

$$
\frac{1}{(\omega+i y)^{2}+1}+\frac{1}{(\omega-i y)^{2}+1}-\frac{1+y}{\omega^{2}+(y+1)^{2}}=\frac{1-y}{\omega^{2}+(1-y)^{2}}
$$

(with $\omega=x-\zeta$ ), we obtain

$$
g(x+i y)+g(x-i y)-\left(P_{y} g\right)(x)=\frac{1}{\pi} \int_{-\infty}^{\infty} \frac{\tau}{(x-\xi)^{2}+\tau^{2}} f(\xi) d \xi,
$$


where $\tau=1-y$. Now as $y \rightarrow 1, \tau \rightarrow 0$, and it is known [2] that the last integral approaches $f(x)$ almost everywhere as $\tau \rightarrow 0$. This proves (10).

\section{BIBLIOGRAPHY}

1. H. Bateman, Some integral equations of potential theory, J. Appl. Phys. 17 (1946), 91-102.

2. H. Pollard, The Poisson transform, Trans. Amer. Math. Soc. 78 (1955), 541550.

Cornell University and

Purdue University

\section{ON HYPONORMAL OPERATORS}

\section{TSUYOSHI ANDÔ}

A bounded linear operator $T$ on a Hilbert space $\mathfrak{F}$ is said to be hyponormal in case $\left\|T^{*} x\right\| \leqq\|T x\|$ for all $x \in \mathfrak{F}$. This short note gives a negative answer to the question raised in $[1$, p. 188]: "Does there exist a completely continuous hyponormal operator which is not normal?"

TheOREM. If $T$ is hyponormal, $\left\|T^{n}\right\|=\|T\|^{n}$ for all $n$.

Proof. It is sufficient to prove that $\|T\|=1$ implies $\left\|T^{n}\right\|=1$ for all $n$. Consider the following property:

$\left(C_{n}\right)$ For every $\epsilon>0$, there exists a unit vector $x$ such that

$$
\left\|T^{n} x\right\| \geqq 1-\epsilon \text { and }\left\|T^{n} x-T^{*} T^{n+1} x\right\| \leqq \epsilon .
$$

$\left(\mathrm{C}_{0}\right)$ just says that 1 is an approximate proper value for the selfadjoint operator $T^{*} T$ (see $\left[1\right.$, p. 170]). $\left(\mathrm{C}_{n}\right)$ obviously implies $\left\|T^{n}\right\|=1$. Now suppose that $\left(\mathrm{C}_{n}\right)$ is valid. For $\epsilon>0$ and $x$ (indicated in $\left.\left(\mathrm{C}_{n}\right)\right)$

$$
\begin{aligned}
&\left\|T^{n+1} x-T^{*} T^{n+2} x\right\|^{2} \\
& \\
&=\left\|T^{n+1} x\right\|^{2}-2\left\|T^{n+2} x\right\|^{2}+\left\|T^{*} T^{n+2} x\right\|^{2} \\
& \leqq\left\|T^{n} x\right\|^{2}-\left\|T^{n+2} x\right\|^{2} \text { (because }\|T\|=\left\|T^{*}\right\|=1 \text { ) } \\
& \leqq\left\|T^{n} x\right\|^{2}-\left\|T^{*} T^{n+1} x\right\|^{2} \text { (because } T \text { is hyponormal) } \\
& \leqq\left\|T^{n} x-T^{*} T^{n+1} x\right\|\left\{\left\|T^{n} x\right\|+\left\|T^{*} T^{n+1} x\right\|\right\} \leqq 2 \epsilon \text { by }\left(\mathrm{C}_{n}\right) .
\end{aligned}
$$

Received by the editors February 12, 1962. 